\title{
A case of chronic necrotizing bronchopulmonary aspergillosis presenting with hemoptysis and a pulmonary mass mimicking lung cancer
}

Servet Kayhan ${ }^{1 *}$, Ünal Şahin ${ }^{1}$, Yıldıray Bekar ${ }^{2}$, Halit Çınarka ${ }^{1}$, Aziz Gümüş ${ }^{1}$, Recep Bedir $^{3}$

1.Department of chest disease, Recep Tayyip Erdogan University, School of Medicine, Rize, Turkey

2.Department of thoracic surgery, Kanuni Research and Training Hospital, Trabzon, Turkey

3.Department of pathology, Recep Tayyip Erdogan University, School of Medicine, Rize, Turkey

\begin{abstract}
Chronic necrotizing pulmonary aspergillosis usually affects the immunocompromised patients with abnormal pulmonary defense mechanisms. Herein, we report a 62-year-old man admitted to our clinic with the complaint of chronic productive cough, hemoptysis and history of recurrent lower respiratory tract infections in last one year. Computed tomographic scan revealed an irregularly shaped mass in the right middle lobe. Fiberoptic bronhoscopy demonstrated the hemorrhage and stenosis in the middle lobe bronchus. Cytologic examinations of bronchoscopic samples demonstrated the hyphae of Aspergillus. An urgent thoracotomy, right middle lobectomy and sleeve resection of posterior segment of upper lobe were performed due to massive hemoptysis and the final diagnosis was local invasive and necrotizing aspergillosis.
\end{abstract}

Keywords: Bronchopulmonary; Aspergillosis; Hemoptysis

\section{Introduction}

Several microorganisms can affect the respiratory system leading to undesirable pulmonary complications (1-4). Despite the advances in diagnostic techniques and treatment modalities, some of the pulmonary pathologies may remain undiagnosed; moreover, recurrences and the complications of the pulmonary infections are also common
(5-8). Among the pathogen germs, fungal agents may cause numerous pathologies in human (9).

As an airborne fungus, Aspergillus is commonly found in the environment. Aspergillus generally develops in immunodeficient patients and causes a variety of clinical syndromes in the lung including

\section{Corresponding author:}

Servet Kayhan, MD

Recep Tayyip Erdogan University, School of Medicine, Department of Chest Disease, 53200 Rize, Turkey Phone +904642123009, email: kayhanservet@gmail.com

Received: 2014-02-17 | Accepted: 2014-03-20 | Published:

2014-04-03 DOI: 10.7575/aiac.abcmed.14.02.02.18 
invasive

aspergillosis,

bronchopulmonary aspergillosis, chronic necrotizing pulmonary aspergillosis (CNPA) and aspergilloma (10-12). CNPA, a different type of invasive aspergillosis first described by Binder et al., shows a subacute clinical table and a relatively local invasive form (13). Radiological presentation of Aspergilloma or mycetoma may be as a pulmonary mass and aspergillosis may mimic the lung cancer.

To the best of our knowledge, there are only a few reports on CNPA mimicking lung cancer without underlying pulmonary lesion $(14,15)$. In this report, we describe a case of CNPA, mimicking lung cancer, manifested with cough and hemoptysis, that appeared in a previously healthy person. Definitive diagnosis was confirmed by surgical removal of the mass.

\section{Case Report}

A 62-year-old man was admitted to our clinic with the complaint of hemoptysis, chronic productive cough and history of frequent antibiotic use due to recurrent lower respiratory tract infections in the last year. On physical examination, the patient was normotensive and afebrile with heart rate of 98 beats/minute. Pulmonary auscultation revealed decreased sounds in the right middle lobe. Laboratory data, consistent with acute/chronic infection, showed a mild anemia and elevated acute phase reactants, white blood cell count: 11500 /mL, hemoglobin: 10.2 g/dL, hematocrit: $30.5 \%$, erythrocyte sedimentation rate 76 $\mathrm{mm} / \mathrm{h}$ and C-reactive protein 1.96 (0-0.8) $\mathrm{mg} / \mathrm{dL}$. Postero-anterior chest radiograph revealed a heterogenous opacity in right paracardiac region and middle zone (Figure-1).

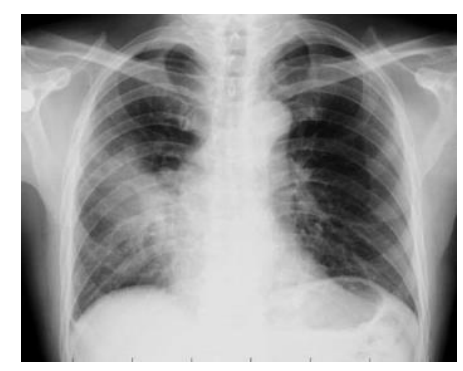

Figure 1: Postero-anterior chest radiograph shows heterogenous opacity in right paracardiac region and middle zone.

Contrast-enhanced computed tomography showed an irregularly shaped non enhanced mass shadow in the right middle lobe (Figure$2 a$ and $2 b)$.

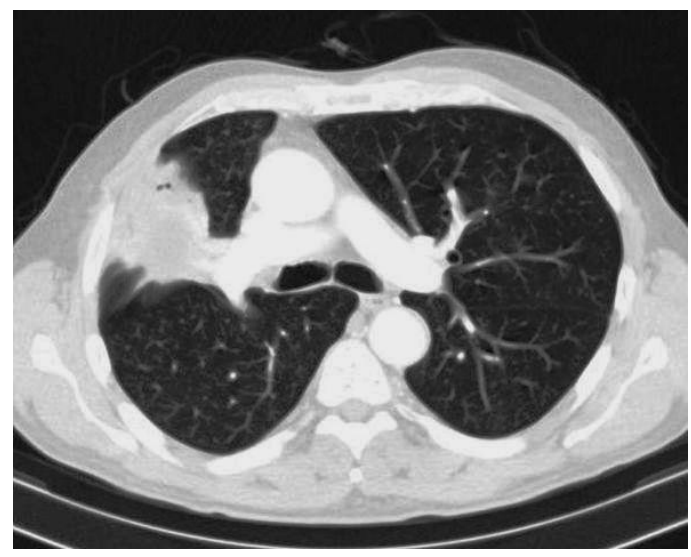

Figure 2a: The parenchymal window of axial computed tomography of the chest reveals a lesion with necrosis and consolidation in right posterior segment of upper lobe and middle lobe.

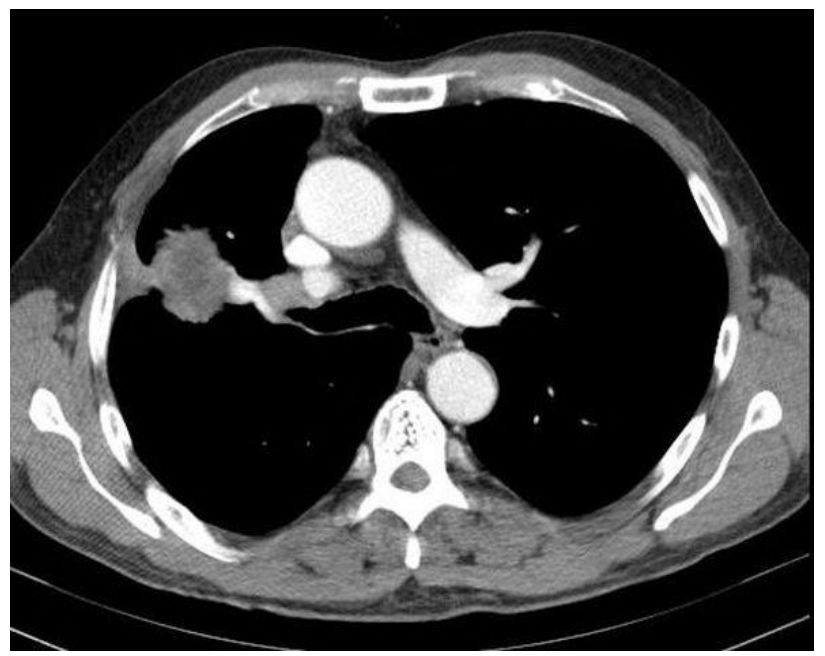

Figure 2b: Contrast enhanced axial computed tomography of chest (mediastinal window) reveals a mass like lesion $(4.7 \times 4.2 \mathrm{~cm})$ extending through the pleural space in right middle lobe with irregular edges.

Fiberoptic bronchoscopy revealed hemorrhage and stenosis in the middle lobe bronchus (Figure-3). Forceps biopsies, bronchial 
brushing and bronchoalveolar lavage were performed. The subsequent bleeding in the middle lobe bronchus was treated and resolved with intrabronchial epinephrine.

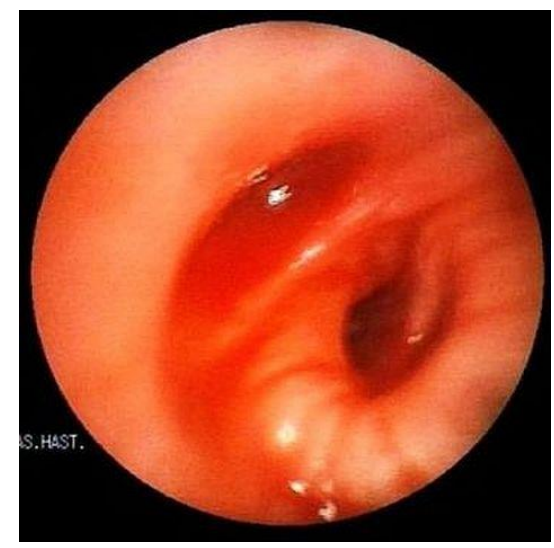

Figure 3: Fiberoptic bronchoscopy reveals active hemorrhage and complete obstruction in right middle lobe.

Cytologic examinations of the bronchoscopic samples demonstrated the hyphae of Aspergillus without any evidence of lung cancer (Figure-4).

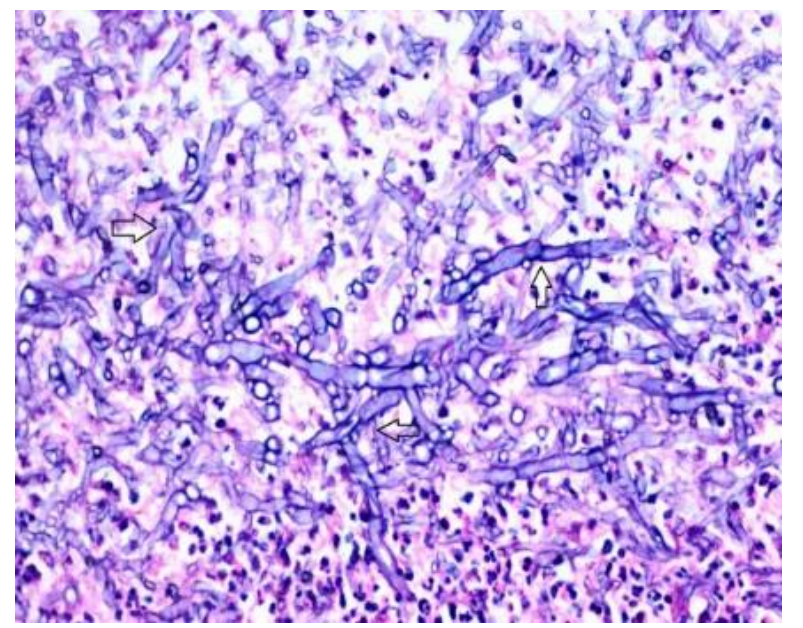

Figure 4: Histopathologic examination showed numerous hyphae branching with approximately 450 angle, characteristic of Aspergillus fumigatus (black arrow) (H\&Ex400)

Oral itraconazole at a daily dose of $400 \mathrm{mg}$ was administered. Positron emission tomography with 2-fluoro (18F)-2-deoxy-Dglucose (FDG) was performed to detect preexisting or concurrent malignancy with aspergillosis. The maximum standard uptake value (SUVmax) was calculated as 6.60 and it was reported that increased metabolic activity in the lesion might be consistent with malignancy. An urgent thoracotomy and right middle lobectomy with sleeve resection of posterior segment of upper lobe was performed following a massive hemoptysis. Postoperative pathological specimens also revealed the diagnosis of chronic necrotizing pulmonary aspergillosis without any evidence of lung cancer (Figure-5).

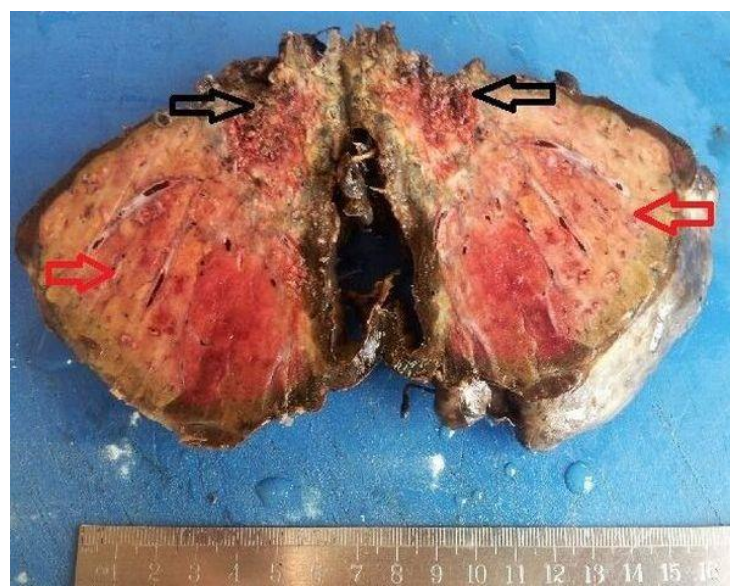

Figure 5: Pathology of the specimen. Macroscopic appearence of the resected tissue $(10.5 \times 10 \times 6 \mathrm{~cm})$ showed chronic necrotizing pulmonary aspergillosis (black arrows) and atelectasis (red arrow).

Antifungal treatment was administered and postoperative chest X-ray showed a complete recovery and compensation of the lung in right hemithorax (Figure-6).

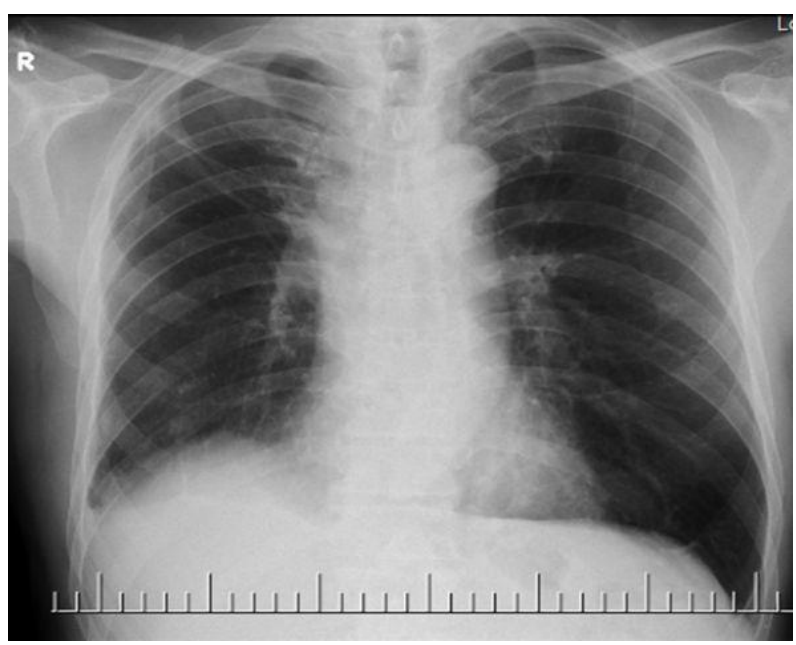


Figure 6: Postoperative postero-anterior chest radiograph of the case reveals complete recovery of the lesions.

\section{Discussion}

Inhalation of Aspergillus conidia may cause various clinical conditions including Aspergilloma, invasive aspergillosis and CNPA depending on the immunological status of the patient (16). A local colonization and growth of fungi without tissue invasion is called as Aspergilloma that usually develops in a preexisting lung cavity arised from tuberculosis, bronchial carcinoma or any other reason (10).

Invasive aspergillosis is a common lifethreatening fungal infections especially among critically ill and intensive care unit patients. The medical equipments, potted plants and water can be a source for hospital acquired infection of Aspergillus (17-19). Allogeneic bone marrow transplantation, intensive chemotherapies, neutropenia, and long-term corticosteroid use are reported as the main predisposing factors for invasive aspergillosis (17).

Another Aspergillus related clinical entity is CNPA which was considered definitive if the compatible symptoms and chest image findings were associated with a positive serum Aspergillus precipitin test or positive isolation of Aspergillus species from a respiratory samples such as sputum or transtracheobronchial aspiration fluid $(11,12,20)$. CNPA is a fungal infectious disease of the lung parenchyma secondary to local invasion by Aspergillus species which affects the immunocompromised patients or hosts with abnormal pulmonary defense mechanisms (21). Our patient presented with the symptoms of chronic productive cough and hemoptysis without any preexisting cavitary lung disease or immunodeficiency and he was treated for pneumonia with multiple rounds of antibiotics.
Serum Aspergillus precipitin antibody tests help with diagnosis and are found to be positive in most of the patients. The frequent chest CT abnormalities of aspergillosis are reported as cavities, parenchymal consolidation, pleural thickening and mycetoma (22). The air crescent sign of mycetoma is more sensitive for diagnosis of Aspergillus which is caused by the existence of crescent of air between mass formation and the inner site of the cavity wall. Our patient represented with a dense consolidation without any cavity in CT which is a nonspecific radiological finding of aspergillosis. We isolated the hyphae of Aspergillus from bronchoscopic bronchial brushing.

CNPA is an uncommon condition and the optimal therapeutic regimen has not been established $(11,12)$. Antifungal therapy with itraconazole or Amphotericin $B$ is the main treatment modality in CNPA (23). Previous studies reported that most of the patients could be safely treated by itraconazole without toxicity $(24,25)$.

On the other hand, despite a long term of antifungal treatment, residual CNPA in pathology specimens was reported by Caras and Pluss after surgery (26). They reported that itrcaconazole treatment without surgery may be only suppressive, rather than curative.

Surgical resection is also needed to treat the complications such as massive hemoptysis and this approach allows for both the definitive pathological diagnosis and treatment concurrently. As we could not wait for the efficacy of long term antifungal treatment, our patient was urgently referred to thoracic surgery due to hemoptysis.

In conclusion, CNPA may mimic a lung cancer and can result in massive hemoptysis leading to a life-threatening disease if left undiagnosed. Therefore, a high level of suspicion is needed to early detection for diagnosis. 


\section{Ethical approval}

The written informed consent of the patient was taken for publication of this case report.

\section{Reference}

1. Sokouti M, Golzari SE, Aghdam BA. Surgery of uncomplicated pulmonary hydatid cysts: capitonnage or uncapitonnage? Int J Surg 2011; 9(3):221-4.

2. Sokouti M. Golzari S. A giant bulla of the lung mimicking tension pnemothorax (a case report). Journal of Cardiovascular and Thoracic Research 2010;2(2):41-44.

3. Sokouti M, Halimi M, Golzari SE. Squamous cell carcinoma on the remaining sequel of tuberculosis, presented as pancoast tumor 8 years later. Tanaffos 2012;11(3):49-51.

4. Kayhan S, Cinarka H, Gumus A, Sahin U. Pneumonia and Pandemic Influenza A H1N1 Virus Infection: A Review of the Literature. ABC med. 2014;2(1):3-14. doi.10/7575/aiac/abcmed/v/2n/1 p/2

5. Golzari SE, Sokouti M, Ghaffari A, Bazzazi AM, Ghabili K. Ultrasonography in diagnosis of pulmonary hydatid cysts. Lancet Infect Dis. 2013;13(4):294.

6. Sokouti M, Pezeshkian M, Ghabili K, Golzari SE. Surgical Procedures and Postoperative Complications in Patients with Giant and Non-giant Pulmonary Hydatid Cysts. Life Sci J 2013;10(1): 138- 142.

7. Feizi I, Sokouti M, Golzari SE, Gojazadeh M, Farahnak MR, Hashemzadeh S, Rahimi-Rad MH. Determination of safe margin in the surgical pathologic specimens of non-small cell carcinoma of the lung. Pneumologia 2013;62(1):16-18.

8. Sokouti M, Golzari SE, Kayhan S, Sabermarouf B. Recurrence Following Pulmonary Hydatid Disease Surgery. World J Surg. 2013 Jul 10.

9. Nejadkazem M, Hemati A, Vegari S, Adibpour M, Davarimajd L. Fungal Agents as a Cause of Nasal Polyposis. ABC med. 2014. (In press)

10. Nam HS, Jeon K, Um SW, Suh GY, Chung MP, Kim H, Kwon OJ, Koh WJ. Clinical characteristics and treatment outcomes of chronic necrotizing pulmonary aspergillosis: a review of 43 cases. Int J Infect Dis. 2010;14(6):e479-82.

11. Saraceno JL, Phelps DT, Ferro TJ, Futerfas R, Schwartz DB. Chronic necrotizing pulmonary aspergillosis: approach to management. Chest 1997;112:541-8.

12. Walsh TJ, Anaissie EJ, Denning DW, Herbrecht R, Kontoyiannis DP, Marr KA, et al. Treatment of aspergillosis: clinical practice guidelines of the Infectious Diseases Society of America. Clin Infect Dis 2008;46:327-60.

13. Binder RE, Faling L, Pugatch RD, Mahasaen C, Snider GL. Chronic necrotizing pulmonary aspergillosis: a discrete clinical entity. Medicine (Baltimore) 1982;61:109-24.

14.Dupont B. Itraconazole therapy in aspergillosis: study in 49 patients. J Am Acad Dermatol 1990;23:607-14.

15.De Beule K, De Doncker P, Cauwenbergh G, Koster M, Legendre R, Blatchford N, et al. The treatment of aspergillosis and aspergilloma with itraconazole, clinical results of an open international study (1982-1987). Mycoses 1988;31:476-85.

16.Caras WE, Pluss JL. Chronic necrotizing pulmonary aspergillosis: pathologic outcome after itraconazole therapy. Mayo Clin Proc 1996;71:25-30.

17. Gefter WB, Weingrad TR, Epstein DM, Ochs RH, Miller WT. 'Semi-invasive' pulmonary aspergillosis: a new look at the spectrum of Aspergillus infections of the lung. Radiology 1981;140:313-21.

18. Binder RE, Faling L, Pugatch RD, Mahasaen C, Snider GL. Chronic necrotizing pulmonary aspergillosis: a discrete clinical entity. Medicine (Baltimore) 1982;61:109-24.

19. Zmeili OS, Soubani AO. Pulmonary aspergillosis: a clinical update. QJM 2007;100:317-34. 
20. Denning DW, Riniotis K, Dobrashian R, Sambatakou H. Chronic cavitary and fibrosing pulmonary and pleural aspergillosis: case series, proposed nomenclature change, and review. Clin Infect Dis 2003;37(Suppl 3):S265-80.

21. Hedayati MT, Mayahi S, Movahedi M, Shokohi T. A Study on Fungal Flora of Tap Water as a Potential Reservoir of Fungi in Hospitals from Sari city, Iran. J Mycol Méd 2011; 21: 10-14.

22. Meersseman W, Vandecasteele SJ, Wilmer A, Verbeken E, Peetermans WE, Van Wijngaerden E. Invasive aspergillosis in critically ill patients without malignancy. Am J Respir Crit Care Med 2004; 170: 621-625.

23. Hedayati MT, Khodavaisy S, Alialy M, Omran SM, Habibi MR. Invasive aspergillosis in intensive care unit patients in Iran. Acta Medica (Hradec Kralove). 2013;56(2):52-6.

24. Takeuchi Y, Shirai T, Sakurai S, Mikamo M, Fujii M, Suda T. Allergic bronchopulmonary aspergillosis presenting with a pulmonary mass mimicking lung cancer. Respirology Case Reports 2013; 1(1): 5-7

25. Yasuda M, Nagashima A, Haro A, Saitoh G. Aspergilloma mimicking a lung cancer. Int J Surg Case Rep. 2013;4(8):690-2. doi: 10.1016/j.ijscr.2013.02.028.

26. Jung SW, Kim MW, Cho SK, Kim HU, Lee DC, Yoon BK, Jeong JP, Ko YC. A Case of Endobronchial Aspergilloma Associated with Foreign Body in Immunocompetent Patient without Underlying Lung Disease. Tuberc Respir Dis (Seoul).2013May;74(5):231-4. 